\title{
A Systematic Review of Reviews of Correctional Mental Health Services Using the STAIR Framework
}

\author{
Alexander I. F. Simpson ${ }^{1 * t}$, Cory Gerritsen ${ }^{2}$, Margaret Maheandiran ${ }^{3}$, Vito Adamo ${ }^{2}$, \\ Tobias Vogel ${ }^{3}$, Lindsay Fulham ${ }^{2}$, Tamsen Kitt ${ }^{4}$, Andrew Forrester ${ }^{5 t}$ and Roland M. Jones ${ }^{2 \dagger}$ \\ ${ }^{1}$ Forensic Psychiatry, Centre for Addiction and Mental Health, University of Toronto, Toronto, ON, Canada, ${ }^{2}$ Department of \\ Forensic Psychiatry, Centre for Addiction and Mental Health, University of Toronto, Toronto, ON, Canada, ${ }^{3}$ Centre for \\ Addiction and Mental Health, Toronto, ON, Canada, ${ }^{4}$ Department of Psychology, Centre for Addiction and Mental Health, \\ University of Toronto, Toronto, ON, Canada, ${ }^{5}$ Forensic Psychiatry, Department of Psychological Medicine and Clinical \\ Neursciences, School of Medicine, Cardiff University, Cardiff, United Kingdom
}

OPEN ACCESS

Edited by:

Xing Liao,

China Academy of Chinese Medical

Sciences, China

Reviewed by:

Jack Tomlin,

University of Greenwich,

United Kingdom

Marije E. Keulen-de Vos,

Forensic Psychiatric Center de

Rooyse Wissel, Netherlands

Keith Reid,

Cumbria, Northumberland Tyne and Wear NHS Foundation Trust,

United Kingdom;

Northumbria University,

United Kingdom

*Correspondence:

Alexander I. F. Simpson

sandy.simpson@camh.ca

tORCID:

Alexander I. F. Simpson orcid.org/0000-0003-0478-2583

Andrew Forrester

orcid.org/0000-0003-2510-1249

Roland M. Jones

orcid.org/0000-0002-3335-4871

Specialty section

This article was submitted to

Forensic Psychiatry,

a section of the journa

Frontiers in Psychiatry

Received: 25 July 2021 Accepted: 13 December 2021

Published: 18 January 2022

Citation:

Simpson AlF, Gerritsen C, Maheandiran M, Adamo V, Vogel T,

Fulham L, Kitt T, Forrester $A$ and Jones RM (2022) A Systematic

Review of Reviews of Correctional Mental Health Services Using the STAIR Framework

Front. Psychiatry 12:747202. doi: 10.3389/fpsyt.2021.747202
Background: Rising demand for correctional mental health services (CMHS) in recent decades has been a global phenomenon. Despite increasing research, there are major gaps in understanding the best models for $\mathrm{CMHS}$ and how to measure their effectiveness, particularly studies that consider the overall care pathways and effectiveness of service responses. The STAIR (Screening, Triage, Assessment, Intervention, and Re-integration) model is an evidence-based framework that defines and measures CMHS as a clinical pathway with a series of measurable, and linked functions.

Method: We conducted a systematic review of the reviews of CMHS elements employing PRISMA guidelines, organized according to STAIR pillars. We assessed the quality of included studies using the AMSTAR-2 criteria. Narrative reviews were read and results synthesized.

Results: We included 26 review articles of which 12 were systematic, metaanalyses, and 14 narrative reviews. Two systematic reviews and seven narrative reviews addressed screening and triage with strong evidence to support specific screening and triage systems. There was no evidence for standardised assessment approaches. Eight systematic reviews and seven narrative reviews addressed interventions providing some evidence to support specific psychosocial interventions. Three systematic reviews and six narrative reviews addressed reintegration themes finding relatively weak evidence to support reintegration methods, with interventions often being jurisdictionally specific and lacking generalizability.

Conclusions: The STAIR framework is a useful way to organize the extant literature. More research is needed on interventions, assessment systems, care pathway evaluations, and reintegration models.

Keywords: prison, systematic review, mental health care, STAIR model, screening

\section{INTRODUCTION}

Rising prison populations internationally have been a source of major concern (1). Although the percentage of prison inmates who have a serious mental illness (SMI) has been relatively static over time at $15 \%$ (2), increasing prison musters mean there are many more people with SMI in custody $(1,3)$. Historically, there has been low access to mental health care in custody and few benchmarks 
to measure the adequacy of services (4). Human rights standards [for instance UNDOC (5) also known as "The Nelson Mandela Rules;" Convention against Torture and Other Cruel, Inhuman or Degrading Treatment or Punishment (6); Convention on the Rights of Persons with Disabilities (7); Council of Europe European Prison Rules (8)] have helped to provide levers to improve care, as has litigation arising from failures of service provision in some jurisdictions (9) (see for instance Brown v. Plata, 563 U.S. 493, 2011). Despite this, actual service delivery and quality of care delivered has generally remained inadequate to level of need $(4,10-13)$.

The key elements of correctional mental health services (CMHS) have been articulated for over 30 years. These elements are proactive case detection and assessment, offering a suitable range of services and reintegration planning $(14,15)$. Steadman et al. (16) first described the need to focus on multiple potential points of engagement or diversion for people with SMI in interaction with the criminal justice system noting key intervention points as being at police arrest, court appearance, remand prison and sentenced prison levels, including re-entry and probation/parole level in the community. This gave rise to conceptual models built along this journey, the most prominent being the Critical Time Intervention (CTI) Model of Draine et al. (17) and Draine and Herman (18) which is a framework providing specific time-based interventions to enhance supports and service provision at key points along this pathway. More recently, Forrester and Hopkin (19) have reviewed CMHS from the perspective of defining these service elements as part of a care pathway. This concept of a pathway or a trajectory for people with SMI is now common (20).

There have been three studies of an overall pathway of care for persons with SMI in correctional facilities in a single jurisdiction (21-23). These studies demonstrate the need for frameworks to address the core service quality issues in correctional mental health care, namely access rates, nature and quality of services delivered, resourcing of clinical teams and management of progression, most particularly between institution and at the point of release.

From work in the UK $(1,24)$, New Zealand $(23,25)$, Canada (4, 15), and Ireland (22) and building on the key elements of CHMS previously articulated, there emerged a consensus around the fundamental elements needed for service delivery in custody. We coined the acronym "STAIR" Model $(1,26)$ to define these elements. STAIR stands for Screening, Triage, Assessment, Intervention, and Re-Integration. The STAIR model also links key clinical functions to epidemiologically derived access and intervention targets, providing benchmarks by which to measure performance. Briefly, the model is as follows.

Screening should be available for all inmates at the point of reception, performed by health staff.

The major disorders being screened for are illnesses such as psychosis, major mood disorders, active suicidality or withdrawal from alcohol or other substances. The rate of positive screens is commonly over $30 \%$ of remand men and near $50 \%$ of remand women (27) allowing a clinical service to evaluate whether the expected rate of positive screen is being achieved.

Triage. Most current screening tools have high false positive rates, so a second stage of evaluation by mental health staff is required, referred to as triage. This is a more detailed assessment of the individual's mental health needs and current level of functioning allowing referral to a next level of primary or secondary care.

Assessment. Positive triage will lead to evaluation by a specialist mental health team, including psychiatric assessment and the development of an individual care plan. It should result in $\sim 15 \%$ of the standing prison population being attached to a specialist mental health team (2).

Intervention. A comprehensive range of culturally competent mental health services is required to respond effectively to the differential levels of presenting illness acuity (e.g., acute or intermediate care for those who are severely or acutely unwell, are suicidal or general prison mental health services for those with more stable conditions).

Re-integration. Planning for community reintegration should begin well in advance of the identified release date, to ensure the continuous delivery of healthcare services and that social care services are engaged. This package of care includes engagement with community mental health services and addressing unmet needs in respect of housing, employment and finance. The provision of transitional clinical support during the period of institutional release is preferred.

The purpose of this paper is to review the extant CMHS literature to assess the current evidence in relation to each of these core service elements. We undertook a systematic review of published review articles of each of the service components of the STAIR model. We aimed to describe the current state of knowledge, highlight areas of good quality evidence and identify gaps in knowledge to inform future research.

\section{METHODS}

\section{Search Strategy}

We performed a systematic review of reviews adhering to PRISMA guidelines as well as those laid out in the Joanna Briggs Institute Manual for Evidence Synthesis (28). Three separate searches were conducted for each of the following STAIR elements: (1) screening, triage, assessment (grouped together given that similar tools are used across these stages), (2) intervention, and (3) reintegration. Search terms were used to specify setting (correctional settings), population (severe mental illness), and study type (review paper) across all searches. Each of the three searches were conducted in MedLine and CINAHL. Each database search employed search terms describing (1) the STAIR component under investigation; (2) the setting (correctional); (3) population specifiers (severe mental healthrelated); and (4) specifiers for article type (reviews). These were combined using "AND" statements and each search was assessed for completeness using a set of pre-selected validation articles. The search was limited to review articles published in English from 1995 up until the search date (end of January, 2020) with no date or geographical restrictions.

The search was supplemented in several ways, given that some expected literature may not be indexed in MedLine or CINAHL. To this end, we also searched the Web of Science Core Collection, the Web of Science Conference Proceedings 


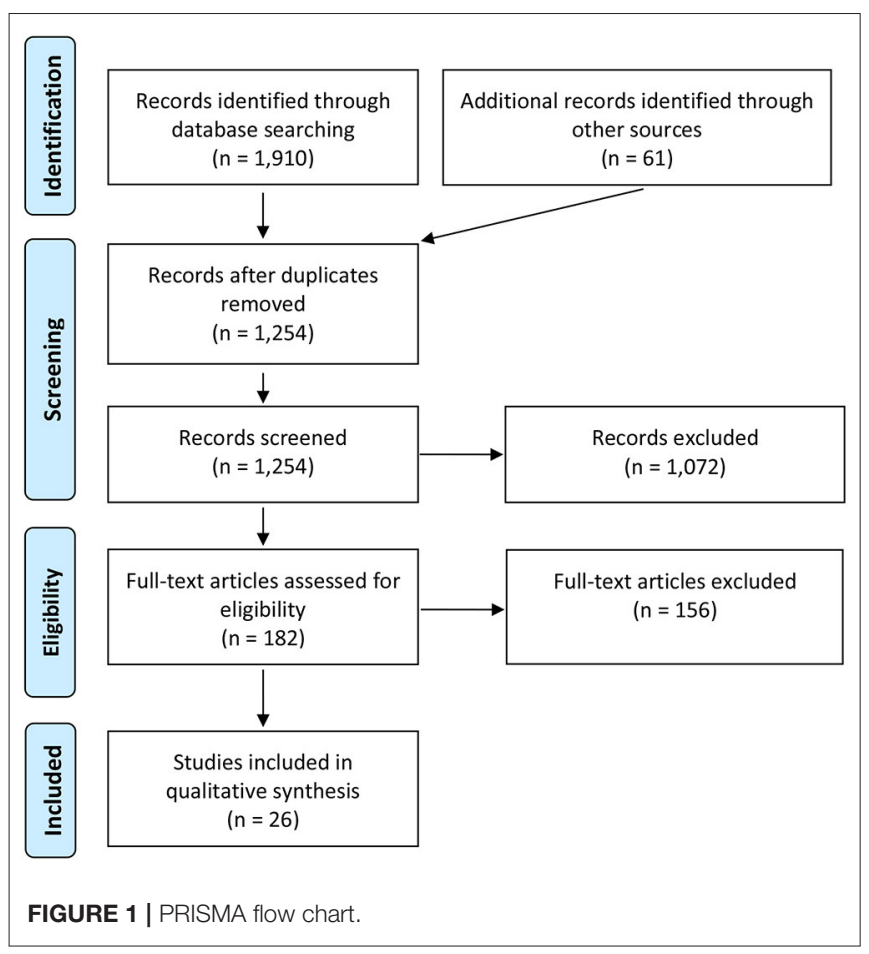

Index, Worldcat/OAlster, and searched government and nongovernmental organization websites. Each of these searches used a condensed set of the terms (the search strategy is attached as Supplementary Materials).

\section{Inclusion Criteria}

We included reviews exploring core CMHS service elements; involving adult prisoners or jail populations with SMI (i.e., psychotic disorder, bipolar disorder, and current severe depressive disorder). Our outcomes of interest were improvement of mental health outcomes broadly (identifying need, reducing symptoms, improving functioning or well-being, accurately identifying SMI). We also applied these criteria to the supplementary searches, except that these were restricted to material that reported data (i.e., opinion papers and unsupported program descriptions were excluded). We excluded papers that (1) provided general discussion or recommendations of services without a review component, or (2) focused only on criminological (e.g., antisocial behavior, recidivism) outcomes among SMI inmates, or outcomes related to suicide or self-harm without specific reference to SMI outcomes, or (3) only focused on substance use or sex offending. Papers with outcome measures that overlapped with those listed above were not excluded. Refer to Figure $\mathbf{1}$ for detailed PRISMA flow chart of the identification, screening, eligibility assessment, and inclusion of articles.

\section{Study Selection}

Two independent reviewers performed title and abstract screening and, where disagreements existed, a third reviewer arbitrated the decision. Finally, the first author (AS) reviewed the selected articles to exclude those that were superseded by a more recent, more comprehensive or higher-quality review in the same content area.

\section{Data Extraction and Evaluation of Quality}

One rater extracted the data into a pre-defined data extraction table (see Table 1), and a second rater confirmed the accuracy of the rating. Any disagreements were resolved by a third rater. The quality of reviews was also evaluated by two raters using the AMSTAR-2 (A MeaSurement Tool used to Assess systematic Reviews; see Table 2) except for non-systematic or narrative reviews which could not meet AMSTAR Criteria (48).

\section{Data Synthesis}

Narrative data synthesis was performed by examining the characteristics and findings of the included reviews, and summarized in the data extraction table. The breadth, quality and consistency of reviewed materials reported were considered in relation to the quality ratings of each review (AMSTAR), and according to clinical and research considerations as adjudged by the current study's authors. Key information pertaining to main findings, study methodology, gaps and future directions were highlighted.

\section{RESULTS}

We identified 26 reviews that met inclusion criteria for the study (see Table 1 for a summary). Seven reviews referenced the need for an integrating model of care as a concept, but there were only two papers that provided primary data regarding models of care $(22,23)$ leaving insufficient comparative data to review overall care pathways.

\section{Screening, Triage, and Assessment: General Overviews and Care Pathways}

Our search found two systematic reviews $(27,37)$ and seven narrative reviews that discussed screening and assessment processes. The methodological quality of the systematic reviews was assessed by AMSTAR 2 and presented in Table 2.

Forrester and Hopkin (19) reviewed pathway models across the criminal justice system with a focus on developments since 2000. Part of their review concerned care in corrections, although they primarily referred to the systematic review of Martin et al. (27). They note that while screening has been a research focus in this area, additional work is needed to ensure coverage of the broad range of disorders seen in correctional settings. The major issue that concerns all individual screening tools is that of poor specificity or the problem of high false positive rates (3). In their narrative review, Kolodziejczak and Sinclair (44) emphasize the need to strike a balance between risks related to over-diagnosis and risks related to under-diagnosis. They note that while under-diagnosis in corrections has clear negative impacts given evidence that many persons with SMI do not access treatment, over-diagnosis has implications for stretching limited health resources which may result in under-attending to those with the most severe needs. Others $(49,50)$ have recommended a two-tiered screening process using a highly sensitive screen on admission to ensure that those in immediate 


\begin{tabular}{llll}
\hline Reference $\quad$ Main focus of review paper & $\begin{array}{l}\text { Elements of STAIR }^{a} \\
\text { AMSTAR score }{ }^{b}\end{array}$ & $\begin{array}{l}\text { Number of } \\
\text { studies in } \\
\text { systematic } \\
\text { review }\end{array}$ \\
\end{tabular}

\section{Systematic Reviews/Meta-analyses $=12$}

Evidence-based strategies for managing suicidal and self-harm behaviors in prisons

Intervention

Deslich (30)

Telepsychiatry in correctional facilities improves access to mental health care and costs

Fontanarosa et al. (31)

Evidence for treatments for offenders with serious mental illness in jail, prison, or forensic hospital, and transitioning from any of these settings to the community

Hopkin et al. (32)

corisoners with mental health conditions that target transition from prison to community

Kendall et al. (33)

Findings from qualita

Compared the sensitivity and specificity of mental health screening tools among adult jail or prison populations

Maruca and Shelton (34)

Summarizes correctional nursing interventions for incarcerated persons with mental disorders

Morgan et al. (35)

Moyes et al. (36)

Treatment effects across studies from service providers to offenders with mental illness

How prison-based services can improve to better meet the needs of prisoners with co-occurring substance misuse and mental health disorders

NICE (37)

This guideline was developed to advise on identification and management of mental health problems and integration of care for adults in contact with the criminal justice system

Smith-Merry et al. (38)

Brings together existing evidence to inform policymakers and practitioners about current practices in transition support, and barriers and facilitators of effective practice

Yoon et al. (39)

列
Intervention

Intervention

Re-integration

Re-integration

Re-integration

Screening

Triage

Assessment

Intervention

Intervention

Intervention

Screening

Triage

Assessment Integration

Re-integration

Re-integration

Intervention mental health outcomes in prisoners and qualitatively summarize difficulties in conducting randomized clinical trials (RCTs)

12

Critically low

Critically low

19 papers

describing 16

studies

Moderate
This review was

supported by the

Queensland Corrective

Services.

None stated

None stated

Self-funded

Health Futures

Development Grant from

the University of

Technology Sydney

None stated

None stated

None stated

None stated

NICE

ner West Partners in

Recovery Flexible funding

Wellcome Trust

(202836/Z/16/Z) 
TABLE 1 | Continued

\begin{tabular}{lll}
\hline Reference $\quad$ Main focus of review paper & $\begin{array}{l}\text { Elements of STAIR }^{\mathrm{a}} \\
\text { Funding reported }\end{array}$ & $\begin{array}{l}\text { Number of } \\
\text { studies in } \\
\text { systematic } \\
\text { review }\end{array}$ \\
\end{tabular}

\section{Narrative Reviews $=14$}

Baillargeon et al. (40)

Reviews challenges to community re-integration among mentally ill prison inmates and promising strategies for improving transition from prison to community

Draine and Herman (18)

Reviews the utility of the Critical Time Intervention (CTI) model, and how to assess its effectiveness

Reviews the utility of the Critical Time Intervention (CT) model, and relevant background research on re-entry and integration

Edens et al. (42)

Fazel et al. (3)

Forrester and Hopkin (19)

Forrester et al. (1)

Jemelka et al. (43)

Kolodziejczak and Sinclair (44)

developed for state and federal prisons in the U.S.

Review of clinical, research, and policy

recommendations to improve mental health care in prisons

Review the nature and extent of evidence streams supporting health care delivery within interagency pathway developments

Reviews issues related to service provision of mental health care in prisons and jails and proposes the utility of the STAIR model

Reviews the issue of mental illness in jails and prisons Includes some treatment and reintegration practices in the U.S. as well as recommendations

Reviews a brief history and overview of mental health services in the U.S. correctional system, as well as a discussion of the barriers to and potential facilitators of providing effective care in the future

Re-integration

Triage

Assessment

Integration

Re-integration

Re-integration

Screening

Triage

Assessment

Intervention Re-integration

Intervention

Triage

Assessment

Intervention

Re-integration

Screening

Triage

Assessment

Intervention

Re-integration

Screening

Triage

Assessment

Intervention

Re-integration

Screening

Triage

Assessment

Intervention

Re-integration
None stated

National Institute of Mental Health (NIMH)

National Institute of Mental Health (NIMH)

None stated

None stated

None stated

None stated

National Institute of Justice

None stated 
TABLE 1 | Continued

\begin{tabular}{|c|c|c|c|c|c|}
\hline Reference & Main focus of review paper & Elements of STAIR ${ }^{a}$ & $\begin{array}{l}\text { Number of } \\
\text { studies in } \\
\text { systematic } \\
\text { review }\end{array}$ & AMSTAR score ${ }^{b}$ & Funding reported \\
\hline Ogloff (15) & $\begin{array}{l}\text { An overview of Canadian-developed correctional and } \\
\text { forensic mental health services to identify and } \\
\text { accommodate the needs of mentally ill people in the } \\
\text { criminal justice system. A six-component model for } \\
\text { mental health services in corrections is advocated in } \\
\text { this report. Covers related issues of diversion from jails } \\
\text { and the need for suicide risk identification and } \\
\text { management in jails. }\end{array}$ & $\begin{array}{l}\text { Screening } \\
\text { Triage } \\
\text { Assessment } \\
\text { Intervention } \\
\text { Re-integration }\end{array}$ & & NA & None stated \\
\hline Peters et al. (45) & $\begin{array}{l}\text { Review of the existing research, examination of key } \\
\text { issues and evidence-based treatment, and supervision } \\
\text { practices related to co-occurring mental and } \\
\text { substance use disorders in the justice system }\end{array}$ & $\begin{array}{l}\text { Screening } \\
\text { Triage } \\
\text { Assessment } \\
\text { Intervention } \\
\text { Re-integration }\end{array}$ & & NA & None stated \\
\hline Simpson et al. (4) & $\begin{array}{l}\text { Reviews the required service components with } \\
\text { particular focus on care models for people with serious } \\
\text { mental illness in the Canadian correctional system }\end{array}$ & $\begin{array}{l}\text { Screening } \\
\text { Triage } \\
\text { Assessment } \\
\text { Intervention } \\
\text { Re-integration }\end{array}$ & & NA & None stated \\
\hline Wallace et al. (46) & $\begin{array}{l}\text { Provides evidence-based and promising treatment } \\
\text { approaches to address the overlap among trauma, } \\
\text { mental illness, substance abuse, and behavioral } \\
\text { problems. A synthesis of research meant to guide } \\
\text { practitioner and policy responses to the national } \\
\text { challenge of meeting the needs of those undergoing } \\
\text { re-entry }\end{array}$ & Re-integration & & NA & $\begin{array}{l}\text { National Institutes of } \\
\text { Health }(\mathrm{NIH})\end{array}$ \\
\hline Winters et al. (47) & $\begin{array}{l}\text { Reviews interventions designed to prevent suicide } \\
\text { among individuals with serious mental illness in } \\
\text { forensic settings, and the need for research to inform } \\
\text { the development of assessment tools and intervention } \\
\text { strategies for this population }\end{array}$ & $\begin{array}{l}\text { Screening } \\
\text { Triage } \\
\text { Assessment } \\
\text { Intervention }\end{array}$ & & NA & None stated \\
\hline
\end{tabular}

aSTA, Screening, Triage, and Assessment; I, Intervention; R, Re-integration; MoC, Model of Care.

${ }^{b} N A$, Not applicable; narrative review articles that were not graded with AMSTAR. 
TABLE 2 | AMSTAR-2 ratings for included systematic reviews and meta-analyses.

\begin{tabular}{|c|c|c|c|c|c|c|c|c|c|c|c|c|c|c|c|c|c|}
\hline \multirow[t]{2}{*}{ References } & \multicolumn{16}{|c|}{ AMSTAR questions $^{a}$} & \multirow[t]{2}{*}{ Overall confidence } \\
\hline & 1 & 2 & 3 & 4 & 5 & 6 & 7 & 8 & 9 & 10 & 11 & 12 & 13 & 14 & 15 & 16 & \\
\hline Barker et al. (29) & Y & $\mathrm{N}$ & Y & PY & $\mathrm{N}$ & $\mathrm{N}$ & $\mathrm{N}$ & Y & $\mathrm{N}$ & $N$ & NM & NM & $\mathrm{N}$ & Y & NM & $\mathrm{N}$ & Critically low \\
\hline Deslich (30) & Y & $N$ & $\mathrm{~N}$ & PY & $\mathrm{N}$ & $\mathrm{N}$ & $\mathrm{N}$ & PY & $\mathrm{N}$ & $\mathrm{N}$ & NM & NM & $\mathrm{N}$ & Y & NM & Y & Critically low \\
\hline Fontanarosa et al. (31) & Y & PY & Y & Y & Y & Y & $\mathrm{Y}$ & $\mathrm{Y}$ & Y & Y & NM & NM & Y & $\mathrm{Y}$ & NM & Y & High \\
\hline Hopkin et al. (32) & Y & PY & Y & Y & Y & $\mathrm{N}$ & PY & PY & Y & Y & NM & NM & $\mathrm{N}$ & $\mathrm{Y}$ & NM & $\mathrm{Y}$ & Moderate \\
\hline Kendall et al. (33) & Y & $\mathrm{N}$ & Y & PY & $\mathrm{N}$ & Y & Y & Y & $\mathrm{N}$ & $\mathrm{N}$ & NM & NM & $\mathrm{N}$ & Y & NM & Y & Moderate \\
\hline Martin et al. (27) & Y & $N$ & Y & PY & $\mathrm{N}$ & Y & PY & PY & Y & $\mathrm{N}$ & NM & NM & $\mathrm{N}$ & $\mathrm{N}$ & NM & Y & Moderate \\
\hline Maruca and Sheldon (34) & Y & PY & Y & PY & $\mathrm{N}$ & $\mathrm{N}$ & $\mathrm{N}$ & $\mathrm{Y}$ & Y & $\mathrm{N}$ & NM & NM & $\mathrm{Y}$ & $\mathrm{Y}$ & NM & Y & Low \\
\hline Morgan et al. (35) & Y & $\mathrm{N}$ & $\mathrm{N}$ & PY & $\mathrm{N}$ & $\mathrm{Y}$ & $\mathrm{N}$ & $\mathrm{Y}$ & Y & $\mathrm{N}$ & Y & $\mathrm{N}$ & $\mathrm{Y}$ & $\mathrm{N}$ & Y & $\mathrm{N}$ & Critically low \\
\hline Moyes et al. (36) & Y & $N$ & $\mathrm{~N}$ & $\mathrm{~N}$ & $\mathrm{~N}$ & $\mathrm{~N}$ & $\mathrm{~N}$ & $\mathrm{~N}$ & $\mathrm{~N}$ & $N$ & NM & NM & $\mathrm{N}$ & $\mathrm{N}$ & NM & $\mathrm{N}$ & Critically low \\
\hline NICE (37) & Y & Y & Y & Y & Y & $\mathrm{N}$ & $\mathrm{Y}$ & $\mathrm{Y}$ & Y & $\mathrm{N}$ & Y & Y & Y & Y & Y & Y & High \\
\hline Smith-Merry et al. (38) & Y & $N$ & Y & PY & Y & $\mathrm{N}$ & $\mathrm{N}$ & $\mathrm{N}$ & $\mathrm{N}$ & $\mathrm{N}$ & NM & NM & $\mathrm{N}$ & Y & NM & Y & Low \\
\hline Yoon et al. (39) & $Y$ & $Y$ & Y & PY & $\mathrm{N}$ & Y & PY & $\mathrm{Y}$ & Y & $\mathrm{N}$ & $Y$ & Y & $\mathrm{Y}$ & $\mathrm{Y}$ & Y & $\mathrm{N}$ & Moderate \\
\hline
\end{tabular}

Y, Yes; PY, Partial Yes; N, No; NM, No meta-analysis conducted.

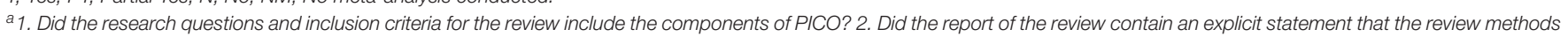

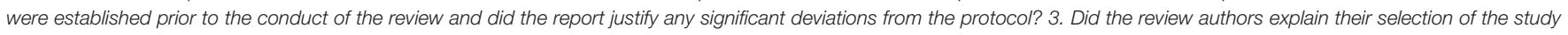

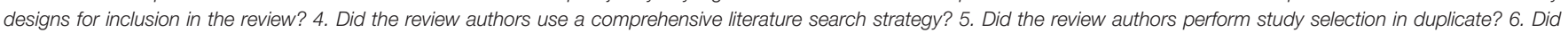

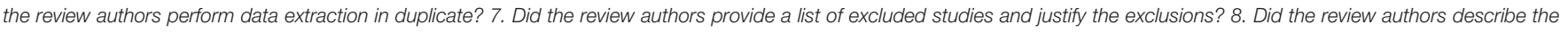

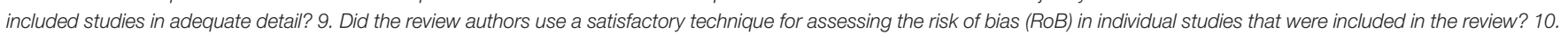

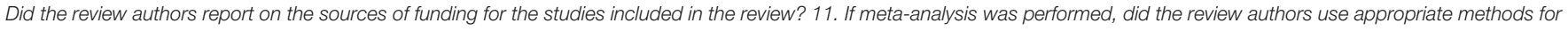

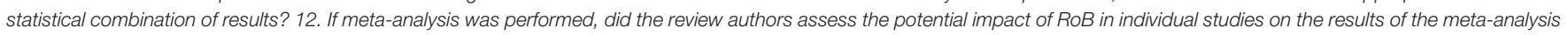

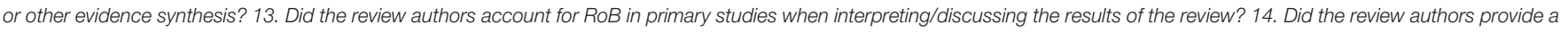

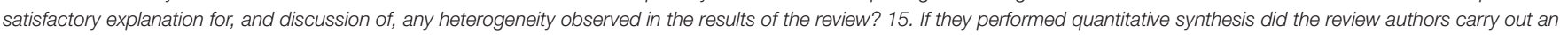

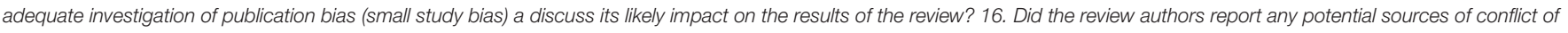
interest, including any funding they received for conducting the review.

need (including those with SMI, or at high risk for self-injury or suicide) are attended to without delay followed by a later, more comprehensive and specific stage that can sort those positive screens into those who do and do not need further mental health assessment. This is essentially the logic of the "ST" component of STAIR (1).

\section{Special Considerations for Comorbid Substance Use in SMI}

Some reviews had a focus on those inmates with comorbid substance use and mental health diagnoses (i.e., concurrent disorders). While the focus of the Moyes et al. (36) review was on treatment, they noted that screening for concurrent disorders was lacking in many facilities. They recommended the integration of concurrent disorder assessment into existing, post-admission visits made by in-reach teams (i.e., during the " $\mathrm{T}$ " or " $\mathrm{A}$ " components of STAIR), given the challenges of performing such assessments upon admission. In contrast, Peters et al. (45) recommended that concurrent disorders be screened at admission, as well as at several other junctures, including initial probation/parole and re-entry points. They note that there are few screening measures that simultaneously address SMI and substance use disorders, and recommend the use of a combination of tools to ensure coverage of both domains.

\section{Screening, Triage, and Assessment: Specific Tools}

Martin et al. (27) performed the seminal systematic review and meta-analysis of screening and triage tools in prison. They identified three screening tools and one triage tool that had robust developmental data and independent validation.

\section{Correctional Mental Health Screen for Women and Correctional Mental Health Screen for Men}

The CMHS-W and CHMS-M are gender-specific tools containing eight and 12 staff-administered items, respectively. Martin et al. (27) cite two studies meeting inclusion criteria; the development paper (51) and a replication by the same authors (52). For the CMHS-W, they found consistent sensitivity rates between the two studies (65 and 64\%, respectively). For the CMHS-M, somewhat higher, similarly consistent sensitivity rates were found between the development and replication studies (74 and $70 \%$, respectively). These two tools have been replicated in a separate sample performed by the original authors with highly consistent findings. QUADAS assessment (26) identified high risk of bias in both of the Ford et al. $(51,52)$ studies with regard to index test use, and applicability concerns in Ford et al. (51) with regard to index test use. The more recent NICE Guideline on the Mental Health of Adults in Contact with the Criminal Justice System (37) did not uncover additional studies on these measures up to 2016.

\section{Brief Jail Mental Health Screen}

The BJMHS (53) is a widely-used staff-rated screening tool. It consists of six symptom-related items and two additional items related to medication and hospitalization. Martin et al. (27) found the original Steadman et al. (53) development article and four additional validation articles; one by the scale's authors (54) 
and three independent studies. The BJMHS was found to show sensitivity of $\sim 60-65 \%$, with the notable exception of one study (55) that yielded a sensitivity of only $34 \%$ among male inmates. When used to screen female inmates, it was found to yield lower sensitivity [e.g., 46\% per Steadman et al. (53)] and may not be considered well-validated for female inmates [as noted in Kubiak et al. (56)]. With regard to rigor, QUADAS ratings were generally positive, with no concerns noted for the Evans et al. (55) study. For three of the studies reviewed $(53,54,57)$, they note risk of bias in patient selection (in addition to risk related to flow and timing in the latter paper), and with regard to Ford et al. (51) they note risk of bias related to the index test used. NICE (37) revealed one additional study (58) yielding an Area Under the Curve (AUC) of 0.72 in a police jail context. NICE appraised the risk of bias in this paper to be high in terms of index test use and reference standard.

\section{England Mental Health Screen}

The EMHS (59) is a four-item tool with a yes/no format, with items focusing on historical factors. A single "yes" response constitutes a positive screen. Martin et al. (27) found four studies including a small pilot, two follow-up studies by teams including the scale's primary author, and one independent study (55). They note that the scale achieved $100 \%$ sensitivity in the pilot (59) but in validation studies involving all-male populations, achieved sensitivities of 42 and $76 \%(55,60)$ In terms of rigor, QUADAS ratings revealed low risk of bias in the original pilot and Evans et al.'s (55) validation, but raised concerns regarding index test selection in the Birmingham et al. (60) study and multiple issues with a study by Gavin et al. (61). The inconsistency in findings across available studies give rise to caution and the potential importance of moderating variables.

\section{Jail Screening Assessment Tool}

The JSAT (15) is a structured professional judgment guide and, as such, requires expertise to administer, having the characteristics of a triage tool, in terms of the STAIR model. It is a semistructured interview lasting $\sim 20 \mathrm{~min}$. It reviews a broad range of factors including mental health issues, current symptoms, substance use, social support, legal situation, and violence. Martin et al. (27) reviewed the original development study (15, 62) and two independent validations (57, 63). Among males, JSAT showed a sensitivity between 38 and $84 \%$, with the latter figure coming from the development study. Among women, it achieved a sensitivity of $75 \%$. Martin et al. (27) highlighted the wide range of findings and interpret this as stemming from the manner in which the JSAT employs structured professional judgment. When a structured scoring cut-off was proposed in one study (57), it yielded a sensitivity of $67-72 \%$. In terms of the rigor of the reviewed studies, QUADAS ratings reflect concerns with bias stemming from patient selection in all studies.

\section{Interventions}

We found eight peer reviewed systematic reviews and seven narrative reviews that discussed interventions within correctional institutions. One gray literature systematic review (37) met our inclusion criteria. The methodological quality of the systematic reviews was assessed by AMSTAR 2 and presented in Table 2.
Kolodziejczak and Sinclair (44) in their narrative review concluded that there is a lack of interventions proven effective for SMI typically available in prisons and noted that, when mental health services are received, they may be limited to medication management due to high caseloads. They nonetheless noted the effectiveness of combined pharmacological and psychotherapeutic approaches, and stressed the importance of addressing comorbid substance use and SMI. They concluded that very little literature specifically evaluates the treatment of SMI within correctional facilities, due to a number of barriers and limitations. Fazel et al. (3) likewise concluded that few studies exist in this area, and those that do tend to be small and yield inconsistent results. A paucity of pharmacological studies was specifically noted.

Yoon et al. (39) conducted an extensive review and metaanalysis of RCTs for psychological interventions in corrections and found a moderate overall effect size of $d=0.50$ across interventions, outcomes and comparators, albeit with large heterogeneity. No difference was found between group and individual administration, but the authors cautioned against assuming equivalence given differences in mean duration between these modalities. Their review yielded seven RCTs with high quality ratings (among the 37 assessed) and found specific support for mindfulness-based and CBT-based interventions, especially for treating depression and anxiety. Martin et al. (64) also conducted a large meta-analysis of interventions designed to reduce criminality or improve mental health in inmates with SMI. They analyzed 25 studies with various modalities, comparators and treatment goals and found evidence for reduced recidivism, better functioning and reduced symptoms across studies. High attrition/rapid turnover, small samples, difficulties in implementing manualized treatments, and loss of effect at follow-up time points were commonly identified.

Some reviews focused on specific treatment modalities, as outlined next.

\section{Pharmacotherapy}

Fazel et al. (3) and Fontanarosa et al. (31) found very few trials for pharmacotherapy in correctional settings, relative to psychological interventions. Fazel et al. (3) included only two, including a trial of ADHD medication improving functioning and promoting abstinence from amphetamine use post-release $(65,66)$ and a trial for a pharmacotherapy decision-making algorithm that resulted in a null finding (67). Fontanarosa et al. (31) reported that evidence is lacking to draw any strong conclusions regarding pharmacotherapy interventions specific to correctional settings; these authors limited their review to trials with active control arms.

\section{Cognitive-Behavioral Therapy}

CBT was, across reviews, the most widely-studied form of psychotherapy in correctional settings. This category included reviewed studies of standard CBT as well as interventions employing CBT principles. Yoon et al. (39) performed the most exhaustive review of CBT among the studies reviewed, and examined CBT separately in their meta-analysis. They found 14 RCTs of CBT with a variety of outcome measures and 
control groups, and concluded that there is moderate-quality evidence supporting this treatment, particularly for anxiety and depression. They did not find evidence supporting the superiority of CBT over other modalities.

\section{Dialectical Behavioral Therapy}

DBT is a highly structured intervention that includes individual psychotherapy (normally 12 months or more), concurrent skills training groups, and structured consult groups for practitioners. Given the challenges of implementing the full DBT model in correctional settings, it is often implemented in an abridged format, and its primary goal has often been the reduction of aggressive incidents (68). Yoon et al. (39) reviewed one RCT with an adequate quality rating, finding a positive but null effect of DBT on trauma and depression symptoms (69). Fazel et al. (3) reviewed a single RCT of DBT for incarcerated women (compared with a shorter-duration DBT regimen plus case management) and found that the former group showed reduced psychopathology.

\section{Interpersonal Therapy}

The reviews by Yoon et al. (39) and NICE (37) uncovered only one RCT of ITP (70). NICE concluded that it provided very low-quality evidence for a clinically significant treatment effect in depression.

\section{Meditation-Based Interventions}

Several current psychotherapies incorporate meditation techniques, such as mindfulness. This category considered approaches based primarily on meditation, including mindfulness-based interventions and Yoga-based interventions. Yoon et al. (39) uncovered five studies in four separate papers examining mindfulness-based interventions in correctional settings, all with risk of bias adequately addressed. They concluded that these therapies were beneficial for symptoms of depression and anxiety. Fazel et al. (3) uncovered one additional, large RCT of a Yoga-based intervention that yielded lower distress and improvements in cognitive function in a prison setting (70). In their review of nursing interventions, Maruca and Shelton (34) additionally found one feasibility study (71) supporting Yoga as a potential treatment for stress and anxiety in incarcerated women.

\section{Trauma-Based/Trauma-Informed Interventions}

Yoon et al. (39) in their systematic review of trauma informed interventions in corrections found six RCTs of therapies classified as trauma-related (including one additional study of cognitive processing therapy, a CBT-based PTSD treatment). Together, the six RCTs failed to achieve statistical significance in metaanalysis. Individual trials that did yield significant effects vs. waitlist or no-treatment controls included Trauma Incident Reduction Therapy (72) Trauma Recovery and Empowerment Model (TREM) for male inmates (73) a brief trauma group (74) and a DBT-based group (69). Two therapies that did not achieve statistical significance were compared to active therapy. NICE (37) reviewed a subset of the same studies; they rated the evidence stemming from the non-null trials reviewed as being of very low to low quality.

\section{Arts-Based Interventions}

NICE (37) reviewed one large RCT of arts-based therapy, yielding very low-quality evidence of clinically significant impact on depression (75). Yoon et al. (39) included this study and three additional trials of art- and music-based therapies, and found that two trials of art-based therapies vs. no treatment, and one out of two trials of music-based therapy vs. an active comparator, yielded positive effects.

\section{Telehealth Interventions}

Deslich (30) reviewed the implementation of telepsychiatry services in correctional settings (vs. in-person services) and found that although telehealth is a platform rather than an intervention, these services improve access without appearing to negatively impact inmate experiences of care, while significantly reducing costs. Fontanarosa et al. (31) cite a prior review by Khalifa et al. (76) suggesting effectiveness of telepsychiatry across multiple forensic settings, including prisons, but note limited outcome-related evidence in this area.

\section{Substance Use and Concurrent Disorders}

Multiple reviews noted the particularly high rate of substance use disorders in those with SMI in incarcerated populations [e.g., up to $80 \%(2)]$, and the importance of simultaneously treating both disorders as per the Integrated Dual Diagnosis Treatment (IDDT) model. This broad framework treats substance and mental health disorders together rather than in parallel or serially, often incorporates intervention models such as CBT and therapeutic community approaches, and yields outcomes superior to approaches targeting either disorder category alone or in parallel $(36,45)$. In their narrative review, Peters et al. (45) noted that given the relatively short time frame of admission to jails, focus should be on acute care, withdrawal management, and community linkage. They found very few studies of injail programs and these tended to be non-integrated and lacking in quantitative data. In terms of prison settings, they found that therapeutic communities (TCs: see the subsection below) had support in comparison with other mental-health focused programming in terms of long-term impact on relapse and re-arrest. Some additional recommendations in this area included the future collection of better-quality evidence, tailoring treatments to gender and stage of change, using peer mentorship, minimizing confrontation and addressing criminogenic thinking $(36,42,45)$.

NICE (37) evaluated several other approaches to substance use disorders, including psychological (e.g., CBT and Acceptance and Commitment Therapy) and pharmacological (e.g., Naltrexone and methadone maintenance) approaches. The majority of these were not specific to SMI populations and examined only substance-related and legal outcomes. The evidence for psychological approaches was of very low to low quality, primarily used active psychological comparison groups and revealed predominantly null findings. They notably examined several RCTs of Naltrexone vs. placebo and found very low-quality evidence of opioid use reduction with Naltrexone treatment. 


\section{Therapeutic Communities}

TCs are milieu-based, interdisciplinary, multifaceted approaches to treating substance use disorders, often incorporating cognitive and behavioral components. Fontanarosa et al. (31) concluded that there is insufficient evidence to judge the comparative effectiveness of TCs and traditional in-prison care for comorbid conditions. NICE (37) uncovered eight RCTs examining TCs and Modified Therapeutic Communities (MTCs) in corrections, yielding very low to low quality evidence for efficacy on a number of psychological symptom and substance use-related indicators, including improvements in substance use for MTCs vs. a CBTinformed group and vs. a traditional mental health program, and mood improvements in an MTC vs. a TC. Several comparisons between TCs, MTCs, and other active control arms in this review were null and considered of very low quality.

\section{Suicidality Interventions}

Winters et al. (47) conducted a review of suicide prevention strategies in SMI populations in corrections and noted that, while CBT, DBT, and IPT programs have shown efficacy in preventing suicide in general settings, these are difficult to implement in corrections, and sparse research exists on corrections-specific programs. They did not find any corrections-specific literature on pharmacological interventions. Barker et al. (29) performed a systematic review of effectiveness literature on suicide and selfharm prevention strategies in prisons, which yielded 12 relevant studies. These were predominantly program implementation studies with $\mathrm{AB}$ designs, and included improved assessment and monitoring, training (notably including the training of peer supporters), special focus on SMI populations and inmates with borderline personality disorder, and review/debriefing strategies. They concluded that such multi-factored interventions focused on mitigating risk factors are particularly effective in reducing suicide outcomes across reviewed studies.

\section{Reintegration}

Reintegration programs focus on the transition period for inmates with SMI who are being released from custody to ensure continuity of their mental health care and other social needs. We found three systematic reviews and six narrative reviews of interventions aimed at transitioning individuals with SMI from custody. Additionally, three gray literature articles met inclusion criteria (see Table 1). The majority of reintegration programs reviewed were from the United States (37) and targeted both the pre- and post-release periods though the actual length of the programs varied widely $(31,32,38)$. The results of the AMSTAR 2 quality assessments of the included reviews are presented in Table 2.

Certain common features of reintegration programs were prerelease planning and post-release support with a combination of practical resources and empathic support (32, 33, 40). These supports can be through remote follow-up or in-person engagement to assist patients having trouble navigating the system (32) and linking them with appropriate community case management (40). This requires trained staff with knowledge of community services. Individualized assessment with a written release plan of the needs and the public safety risk of the inmate
(40) are also crucial to avoiding gaps in treatment. Different approaches are required for remand or pre-trial populations due to the shorter term stays and more unpredictable discharge requiring the assessment of needs to be fast-tracked (17). Traditionally, the goal of re-entry has been to reintegrate the individual into the community with the focus of protecting the community from future harm (41) as opposed to the recoveryoriented and patient centered care that is now the industry standard for mental health services generally (38).

Outcome measures commonly employed included health outcomes such as service use, hospitalizations and medication adherence, and criminal justice outcomes such as reoffending and reincarceration. Severity of symptoms of SMI were rarely used as an outcome measure. Only four of the reviews employed evidence quality assessments in their review methodology (31$33,37)$. Lack of blinding was the biggest issue for weak studies (32).

Effectiveness of programs using criminal justice outcomes was assessed in several studies with only one reporting a significant reduction in reoffending and reincarceration (77) though the evidence was weak due to factors including selection bias and confounders. The evidence for research on other programs were rated of low to high quality. The two studies rated as high reported a non-significant reduction in re-arrests (78) and an increase in reincarceration, respectively (79). Hopkin et al. (32) posited that the increased monitoring offered by the reintegration program may serve as a possible explanation for the increased reincarceration. Studies assessing mental health outcomes were also reported to be of varying quality. IDDT programs that reported reduced psychiatric hospitalizations and mental health service use were of low quality $(31,37)$ with insufficient evidence for impact on substance abuse (31). Research on other interventions reported significantly higher mental health service contacts than the comparator groups and were assessed by Hopkin et al. (32) to be of moderate to high quality.

Three of the trials reviewed by Fontanarosa et al. (31) were conducted in urban areas making it not transferrable to rural areas where community resources may be scarce. Evidence for the impact of specialist vs. mental health generalist care on psychiatric symptoms, psychiatric hospitalization, substance abuse, quality of life, and completed suicide was insufficient as only one trial reported these outcomes (31). The same authors also reported an RCT on Interpersonal therapy (IPT) demonstrating reduction in depressive symptoms but no change to substance-abuse relapse with low risk of bias though this was on the only study on this program and thus insufficient to draw conclusions. A more recent qualitative study of this program also reported program satisfaction with high quality (33).

Multiple barriers to reintegration have been described including lack of funding $(38,40)$ complex post-release care pathways, the need for greater direct service connectivity, insufficient planning resources, a lack of collaboration between correctional facilities and the community and unavailability of medication at release (38). Additionally, the chaotic nature of release particularly for remand inmates may limit the ability of community services to respond to referrals (19). Programs including CTI (80) may not be feasible in rural or 
regional settings where community mental health resources are scarce (38). Unconditional releases (without parole and mandated treatment) pose the most difficult challenges with transitional planning (40). This may be due to difficulties accessing inmates for their participation in re-entry support and lack of participation may reflect concerns and motivations that are independent of the need or desire for mental health care (38).

The majority of reintegration programs fell into the following categories:

1. Bridging plus assistance with benefits application: Programs reviewed were specific to the US where $90 \%$ of jurisdictions terminate or suspend Medicaid upon incarceration and lack of affordable healthcare may mean that many inmates need benefits to continue accessing care upon release (38). The bureaucracy involved in reinstating benefits may impede those with SMI. Transition planning teams have been shown to improve post-release benefit enrolment (40) but the impact on improving mental health outcomes is unclear with limited evidence reported for future contact with the mental health system $(31,32,38)$.

2. ACT programs: Adapted ACT programs ensuring ongoing care for individuals leaving custody is common. One RCT measuring psychological and clinical outcomes demonstrated no significant difference between ACT, forensic caseworkers and treatment as usual (32). Another program used an ACT model to pair probation officers with mental health workers for persons with comorbid SMI and substance use showing less likelihood of re-incarceration though these results were not significant $(32,78)$.

3. Critical time intervention (CTI) and short-term bridging: CTIs for transition support are focused, time-limited interventions that aim to develop an individualized housing, education and employment strategy to increase social inclusion. Such programs are designed to be short-term and connect individuals with community care $(18,38)$. These programs are less effective in areas where community resources are scarce and not feasible unless the case manager is located in the correctional facility (38). In the UK, an RCT on CTI (4 weeks pre and 6 weeks post-release) demonstrated significantly higher registration with a general practitioner ( 87 vs. $38 \% ; p=0.01$ ) and medication administration (80 vs. $38 \% ; p=0.03$ ) although the results lack sufficient power due to the high attrition rate $(19,32)$. A larger RCT by Shaw et al. (81) found that CTI significantly improved engagement with community mental health services at 6 weeks (53 vs. $27 \%, p=$ $0.012)$ and this was maintained at a later follow up 6 months $(p=0.029)$ after release $(19,32)$.

With regard to co-occurring substance use, while some reintegration programs addressed substance use together with SMI (32) re-entry services were often fragmented and were only focused on mental health issues and not sufficient to address other risk factors for criminal recidivism which may not be a result of mental health symptoms (45). Advances have been made in co-occurring disorders (CODs) treatment but such programs are still absent in many communities and correctional facilities (45). IDDT programs shows promise for reducing hospitalization post-release but replication studies are needed (31). Services integrating mental health and substance misuse services should be delivered by staff who have expertise in both areas rather than sequentially or in parallel (45). There are few studies on CODs programs targeted toward female offenders (45). In their review of qualitative studies, Kendall et al. (33) reviewed one such study on female inmates with SMI noting that women valued continuity of care with the same worker.

\section{DISCUSSION}

The needs of persons with SMI in correctional settings remain of major concern. In this review of the reviews of correctional mental health care elements, we set out to describe the state of knowledge of the span of the care pathway during incarceration. To do this we used the organizing structure of the STAIR model to define the key service domains of this care trajectory to enable us to evaluate the strength of knowledge at each step.

We found a very significant number of reviews. However, many were narrative in form and, whilst informative and containing much wisdom about the development and implementation of correctional mental health services, are limited in their generalizability because of the lack of empirical studies upon which to base their guidance. We found 12 systematic reviews or meta-analyses that focused on the domains of screening, interventions and re-entry programs. The areas of greatest knowledge are in screening and triage, psychological therapies and aspects of reintegration in certain jurisdictions.

In the screening and triage area, there are two high quality systematic reviews of multiple tools with independent validation studies. This evidence is sufficient to make recommendations for service design using two screening tools of adequate psychometric integrity (BJMHS and CMHS) and one triage tool (JSAT). Both independently validated screening tools have problems of high false positive rates necessitating triage processes if they are employed in settings with large numbers of persons to be screened. The JSAT is the only validated longer form assessment tool that may be appropriate for the triage of persons referred on the basis of shorter tools such as the BJMHS or CMHS. Proper staff training in the JSAT is crucial given evidence for wide variability in performance across settings in this largely subjectively-rated instrument, while screening tools offer more consistent results and can be administered by non-specialists. These tools remain poorly validated for women, and for those of minority ethnicity. All of the reviews in the S-T-A area focused on measures that are typically implemented in the $S$ and $T$ stages of STAIR. There is a lack of evidence concerning in-depth assessment tools and processes in these populations.

There were eight systematic reviews of interventions, with a sufficient number of robust studies for meta-analyses of some psychosocial interventions. There were few studies of biological interventions in custody. This limited research base in the specific context of correctional facilities may reflect the assumption that the effectiveness of pharmacotherapy interventions for specific disorders are reasonably generalizable from trials of similar patient groups in other settings. The same may well not be 
true for psychological interventions, which may be more heavily moderated by contextual and population-specific factors, and often require modification in correctional settings. There is thus less need to replicate efficacy studies of psychotropic medications in custody than there is a need to demonstrate the efficacy of psych-social interventions. For instance, an effective intervention such as DBT requires significant modification for correctional settings (67) meaning specific trials are needed to demonstrate effusiveness of the modified intervention specific to the mental health and environmental challenges of living in custody. Study in this area is challenging, given setting- and duration-related restrictions. There is now a solid body of evidence for CBT for anxiety and depressive symptoms for persons in custody, whereas sparse or low-quality evidence supports the efficacy of other modalities and the psychological treatment of other presenting problems. Feasibility studies, on the other hand, appear common in this area and support the application of modified forms of several psychological therapies in corrections. Heterogeneity and inconsistent findings are the norm in this field, suggesting that the examination of modifying factors might be a fruitful avenue for future research. Telehealth also appears to be a promising delivery mode for psychotherapy, with early support for non-inferiority and feasibility; this could reduce access barriers in many correctional settings, including for those in segregation.

Reintegration remains a major transition point where particular models of interventions are required to achieve continuity of care for those with SMI to reduce relapse and recidivism (18). To ensure that help is not misplaced, there is a need for individualized post-release plans to address prisoners' unique needs $(38,40)$; prisoners may view mental health needs as secondary to economic considerations such as obtaining housing and employment (82). Programs such as Housing First that aim to address inmates' economic needs have showed weak evidence (20).

The body of evidence for reintegration studies is significant but often limited in generalisability because the studies address jurisdictionally-specific issues such as Medicaid enrollment. Though countries such as Canada, the UK, Australia, and New Zealand have public healthcare, inmates may still need support with drug plan applications to ensure continued access to medications such as antipsychotics necessary for managing symptoms and preventing recidivism (83-85). There is a crucial need for more studies addressing comprehensive support models at the point of release that address social determinants of health (benefits, housing) as well as health and criminogenic issues. The problem of rapid re-incarceration of many persons with SMI being released from custody (86) underlines this need. While the purpose of reintegration has shifted from protecting the community from future harm to addressing the inmates' recovery needs, only one study assessed symptom improvement as an outcome (38). Among people with severe mental illness, incarceration is five times more likely among those with a co-occurring substance use disorder $(38,87)$ yet few re-entry programs were aimed at substance abuse.
Few reintegration studies were specific to women, though research has shown that woman have different demographic, health, and criminal characteristics (3). Factors such as women being more likely to have children will impact their reintegration needs. There were no studies of reintegration of aboriginal populations or other racialized minorities that are overrepresented among incarcerated populations. There was a lack of studies assessing re-entry programs in middle and low income countries despite higher rates of SMI amongst their prison populations (2). Community reintegration programs need effective community mental health care to pick up the care of the person exiting custody. Lower income, marginalized neighborhoods having disproportionately higher numbers of the incarcerated individuals where the scarcity of community mental health resources may result in a cycle of reincarceration (88). Bridging programs may also be particularly challenging in countries that have large regional, rural and remote areas such as Australia (38).

We also found areas of weakness. Whilst the screening tools are well-studied, all have problems with high false positive rates; there are few studies of cross gender effectiveness and cross-cultural effectiveness. Given that persons of minority ethnicity are over-represented in custody, ensuring tools are effective for the particular ethnic groups in a jurisdiction remains a challenge that has been rarely addressed. Second, there are no studies of standardized assessment tools of severity of illness measures in routine use, both to describe need at point of service entry and as measures of effectiveness of interventions or systems of care. The Clinical Global Imopression-Corrections (CGI-C) scale is one promising such tool that has been validated in Canada and Germany (86, 89). There are few studies of the overall care pathway, the studies of O'Neill et al. (22) and Pillai et al. (23) being notable exceptions. More studies of this type are needed linking service provision to quality indicators at multiple points across the care trajectory. The systematic review of intervention studies found too few intervention studies to inform services of effective intervention approaches.

We employed the STAIR model to organize this literature and found it a helpful framework to show areas of strength and areas of weakness in existing research in each area. The principle of seeing CMHS as an integrated care pathway, with measurable levels of access and expected quality outcomes, is crucial to focusing forensic research and delivery initiatives to improve service outcomes.

\section{Limitations}

The major limitation is the diversity of the literature, and too few studies in a number of areas to come to clear recommendations about evidence-based recommendations. As we chose to only review reviews, there may be primary studies in some areas and smaller studies that are in need of replication that we have not included. There may be promising practices in these excluded studies that need to be more rigorously tested in an experimental paradigm. 


\section{CONCLUSION}

There is a rich literature in correctional mental health services with some areas of strength but other areas of weakness. The STAIR model provides a framework to organize our thinking about these needs and to focus more research on care pathways and performance measures. New research is needed into therapeutic interventions and reintegration needs in particular.

\section{DATA AVAILABILITY STATEMENT}

The original contributions presented in the study are included in the article/Supplementary Material,

\section{REFERENCES}

1. Forrester A, Till A, Simpson A, Shaw J. Mental illness and the provision of mental health services in prisons. Br Med Bull. (2018) 127:1019. doi: 10.1093/bmb/ldy027

2. Fazel S, Seewald K. Severe mental illness in 33588 prisoners worldwide: systematic review and meta-regression analysis. Br J Psychiatry. (2012) 200:364-73. doi: 10.1192/bjp.bp.111.0 96370

3. Fazel S, Hayes AJ, Bartellas K, Clerici M, Trestman R. Mental health of prisoners: prevalence, adverse outcomes, and interventions. Lancet Psychiatry. (2016) 3:871-81. doi: 10.1016/S2215-0366(16)3 0142-0

4. Simpson AI, McMaster JJ, Cohen SN. Challenges for Canada in meeting the needs of persons with serious mental illness in prison. J Am Acad Psychiatry Law Online. (2013) 41:501-9.

5. UNDOC. United Nations Standard Minimum Rules for the Treatment of Prisoners (the Nelson Mandela Rules). A/RES/70/175. (2015) General Assembly, Vienna, Justice Section, UNDOC. Available Online at: https://www. un.org/en/events/mandeladay/mandela_rules.shtml

6. UNDOC. Torture and other InHuman or Degrading Treatment or Punishment. A/36/426. (1981) Genera Assembly, Vienna.

7. UNDOC. Convention on the Rights of Persons with Disabilities. A/RES/61/106. (2006) General Assembly, Vienna.

8. Council of Europe. European Prison Rules. Strasbourg: Council of Europe Publishing (2006).

9. Forrester A, Piper M. The WPA's prison health position statement and curriculum. World Psychiatry. (2020) 19:125. doi: 10.1002/wps.20711

10. Foulds JA, Monasterio E. A public health catastrophe looms: the Australian and New Zealand prison crisis. Austr N Zeal J Psychiatry. (2018) 52:101920. doi: 10.1177/0004867418802902

11. Skipworth J. The Australian and New Zealand prison crisis: cultural and clinical issues. Austr $N$ Zeal J Psychiatry. (2018) 53:472-3. doi: 10.1177/0004867418817375

12. Simpson AIF, Jones RM. Two challenges affecting access to care for inmates with serious mental illness: detecting illness and acceptable services. Can J Psychiatry. (2018) 63:648-50. doi: 10.1177/07067437187 92844

13. Piper M, Forrester A, Shaw J. Prison healthcare services: the need for political courage. Br J Psychiatry. (2019) 215:579-81. doi: 10.1192/bjp.2019.43

14. APA. Treatments of Psychiatric Disorders: A Task Force Report of the American Psychiatric Association. Washington, DC: American Psychiatric Association (1989).

15. Ogloff JR. Identifying and accommodating the needs of mentally ill people in gaols and prisons. Psychiatry Psychol law. (2002) 9:1-33. doi: 10.1375/pplt.2002.9.1.1 further inquiries can be directed to the corresponding author/s.

\section{AUTHOR CONTRIBUTIONS}

AS conceived of the review. CG, MM, VA, TV, LF, and TK performed review, the data extraction, and quality ratings. MM, CG, AS, AF, and RJ drafted the manuscript. All authors contributed to the article and approved the submitted version.

\section{SUPPLEMENTARY MATERIAL}

The Supplementary Material for this article can be found online at: https://www.frontiersin.org/articles/10.3389/fpsyt. 2021.747202/full\#supplementary-material

16. Steadman HJ, McCarty DW, Morrissey JP. The Mentally Ill in Jail: Planning for Essential Services. New York, NY: Guilford Press (1989).

17. Draine J, Blank A, Kottsieper P, Solomon P. Contrasting jail diversion and injail services for mental illness and substance abuse: do they serve the same clients? Behav Sci Law. (2005) 23:171-81. doi: 10.1002/bsl.637

18. Draine J, Herman DB. Critical time intervention for reentry from prison for persons with mental illness. Psychiatric Services. (2007) 58:157781. doi: 10.1176/ps.2007.58.12.1577

19. Forrester A, Hopkin G. Mental health in the criminal justice system: a pathways approach to service and research design. Crim Behav Mental Health. (2019) 29:207-17. doi: 10.1002/cbm.2128

20. NICE. Health of People in the Criminal Justice System NICE Pathways. (2019). Available online at: https://pathways.nice.org.uk/pathways/health-of-peoplein-the-criminal-justice-system/health-of-people-in-the-criminal-justicesystem-overview (accessed December 20, 2021).

21. Ogloff JR, Tien G, Roesch R, Eaves D, A. model for the provision of jail mental health services: an integrative, community-based approach. J Mental Health Admin. (1991) 18:209-22. doi: 10.1007/BF02518592

22. O'Neill C, Smith D, Caddow M, Duffy F, Hickey P, Fitzpatrick M, et al. STRESS-testing clinical activity and outcomes for a combined prison in-reach and court liaison service: a 3-year observational study of 6177 consecutive male remands. Int J Mental Health Syst. (2016) 10:97. doi: 10.1186/s13033-016-0097-z

23. Pillai K, Rouse P, McKenna B, Skipworth J, Cavney J, Tapsell R, et al. From positive screen to engagement in treatment: a preliminary study of the impact of a new model of care for prisoners with serious mental illness. BMC Psychiatry. (2016) 16:1-7. doi: 10.1186/s12888-016-0711-2

24. OHRN. The Pathway of Prisoners With MH Problems Through Prison Health Services. Manchester (2010).

25. McKenna B, Skipworth J, Tapsell R, Pillai K, Madell D, Simpson A, et al. Impact of an assertive community treatment model of care on the treatment of prisoners with a serious mental illness. Austral Psychiatry. (2018) 26:2859. doi: $10.1177 / 1039856217748247$

26. Nicholls TL, Butler A, Kendrick-Koch L, Brink J, Jones R, Simpson AIF. Assessing and treating offenders with mental illness. In: Ternes M, Magaletta PR, Patry MW, editors, The Practice of Correctional Psychology. Cham: Springer International Publishing (2018). p. 9-37. doi: 10.1007/978-3-030-00452-1_2

27. Martin MS, Colman I, Simpson AI, McKenzie K. Mental health screening tools in correctional institutions: a systematic review. BMC Psychiatry. (2013) 13:1-10. doi: 10.1186/1471-244X-13-275

28. Aromataris E, Munn Z. JBI Manual for Evidence Synthesis. JBI, 2020. Available Online at: https://synthesismanual.jbi.global

29. Barker E, Kõlves K, De Leo D. Management of suicidal and self-harming behaviors in prisons: systematic literature review of evidence-based activities. Archiv Suicide Res. (2014) 18:227-40. doi: 10.1080/13811118.2013.824830 
30. Deslich S. Telepsychiatry in correctional facilities: using technology to improve access and decrease costs of mental health care in underserved populations. Perm J. (2013) 17:80-6. doi: 10.7812/TPP/12-123

31. Fontanarosa J, Uhl S, Oyesanmi O, Schoelles KM. Interventions for Adult OffendersWith Serious Mental Illness. Report No.: 13-EHC107. Maryland, ML: Agency for Healthcare Research and Quality (US) (2013).

32. Hopkin G, Evans-Lacko S, Forrester A, Shaw J, Thornicroft G. Interventions at the transition from prison to the community for prisoners with mental illness: a systematic review. Admin Pol Mental Health Mental Health Serv Res. (2018) 45:623-34. doi: 10.1007/s10488-018-0848-z

33. Kendall S, Redshaw S, Ward S, Wayland S, Sullivan E. Systematic review of qualitative evaluations of reentry programs addressing problematic drug use and mental health disorders amongst people transitioning from prison to communities. Health Justice. (2018) 6:8. doi: 10.1186/s40352-018-0063-8

34. Maruca AT, Shelton D. Correctional nursing interventions for incarcerated persons with mental disorders: an integrative review. Issues Ment Health Nurs. (2016) 37:285-92. doi: 10.3109/01612840.2016.1145308

35. Morgan RD, Flora DB, Kroner DG, Mills JF, Varghese F, Steffan JS. Treating offenders with mental illness: a research synthesis. Law Hum Behav. (2012) 36:37-50. doi: 10.1037/h0093964

36. Moyes HCA, Nat Wright DPW, Heath JJ, Dean LV. What can be done to improve outcomes for prisoners with a dual diagnosis? Adv Dual Diagn. (2016) 9:14-25. doi: 10.1108/ADD-07-2015-0016

37. NICE. Mental Health of Adults in Contact With the Criminal Justice System NICE Pathways. (2017). Available online at: https://pathways.nice.org. uk/pathways/health-of-people-in-the-criminal-justice-system/health-ofpeople-in-the-criminal-justice- system-overview\#path=view\%3A/pathways/ health-of-people-in-the-criminal-justice-system/managing-the-mentalhealth-of-people-in-the-criminal-justice-system.xml\&content=view-index (accessed December 20, 2021).

38. Smith-Merry J, Goggin G, Campbell A, McKenzie K, Ridout B, Baylosis C. Social connection and online engagement: insights from interviews with users of a mental health online forum. JMIR Ment Health. (2019) 6:e11084. doi: 10.2196/11084

39. Yoon IA, Slade K, Fazel S. Outcomes of psychological therapies for prisoners with mental health problems: a systematic review and meta-analysis. J Consult Clin Psychol. (2017) 85:783-802. doi: 10.1037/ccp0000214

40. Baillargeon J, Hoge SK, Penn JV. Addressing the challenge of community reentry among released inmates with serious mental illness. Am J Community Psychol. (2010) 46:361-75. doi: 10.1007/s10464-010-9345-6

41. Draine J, Wolff N, Jacoby JE, Hartwell S, Duclos C. Understanding community re-entry of former prisoners with mental illness: a conceptual model to guide new research. Behav Sci Law. (2005) 23:689-707. doi: 10.1002/bsl.642

42. Edens JF, Peters RH, Hills HA. Treating prison inmates with co-occurring disorders: an integrative review of existing programs. Behav Sci Law. (1997) 15:439-57. doi: 10.1002/(SICI)1099-0798(199723/09)15:4<439::AIDBSL282>3.0.CO;2-X

43. Jemelka R, Trupin E, Chiles JA. The mentally ill in prisons: a review. Hosp Commun Psychiatry. (1989) 40:481-91. doi: 10.1176/ps.40.5.481

44. Kolodziejczak O, Sinclair SJ. Barriers and facilitators to effective mental health care in correctional settings. J Correction Health Care. (2018) 24:25363. doi: $10.1177 / 1078345818781566$

45. Peters RH, Young MS, Rojas EC, Gorey CM. Evidence-based treatment and supervision practices for co-occurring mental and substance use disorders in the criminal justice system. Am J Drug Alcohol Abuse. (2017) 43:47588. doi: 10.1080/00952990.2017.1303838

46. Wallace BC, Conner LC, Dass-Brailsford P. Integrated trauma treatment in correctional health care and community-based treatment upon reentry. $J$ Correct Health Care. (2011) 17:329-43. doi: 10.1177/1078345811413091

47. Winters GM, Greene-Colozzi E, Jeglic EL. Preventing suicide in forensic settings. J Correction Health Care. (2017) 23:38397. doi: 10.1177/1078345817725047

48. Shea BJ, Reeves BC, Wells G, Thuku M, Hamel C, Moran J, et al. AMSTAR 2: a critical appraisal tool for systematic reviews that include randomised or non-randomised studies of healthcare interventions, or both. BMJ. (2017) 358:j4008. doi: 10.1136/bmj.j4008

49. Hassan L, Rahman MS, King C, Senior J, Shaw J. Level of mental health intervention and clinical need among inmates with mental illness in five english jails. Psychiatric Serv. (2012) 63:1218-24. doi: 10.1176/appi.ps.201100344

50. Martin K, Martin E. Factors influencing treatment team recommendations to review tribunals for forensic psychiatric patients. Behav Sci Law. (2016) 2016:2244. doi: 10.1002/bsl.2244

51. Ford JD, Trestman RL, Wiesbrock V, Wanli Z. Development and validation of a brief mental health screening instrument for newly incarcerated adults. Assessment. (2007) 14:279-99. doi: 10.1177/1073191107302944

52. Ford JD, Trestman RL, Wiesbrock VH, Zhang W. Validation of a brief screening instrument for identifying psychiatric disorders among newly incarcerated adults. Psychiatr Serv. (2009) 60:842-6. doi: 10.1176/ps.2009.60.6.842

53. Steadman HJ, Scott JE, Osher F, Agnese TK, Robbins PC. Validation of the brief jail mental health screen. Psychiatr Serv. (2005) 56:81622. doi: 10.1176/appi.ps.56.7.816

54. Steadman HJ, Robbins PC, Islam T, Osher FC. Revalidating the brief jail mental health screen to increase accuracy for women. Psychiatr Serv. (2007) 58:1598-601. doi: 10.1176/ps.2007.58.12.1598

55. Evans C, Brinded P, Simpson AI, Frampton C, Mulder RT. Validation of brief screening tools for mental disorders among New Zealand prisoners. Psychiatr Serv. (2010) 61:923-8. doi: 10.1176/ps.2010.61.9.923

56. Kubiak SP, Beeble ML, Bybee D. Using the K6 to assess the mental health of jailed women. J Offender Rehabil. (2009) 48:296-313. doi: 10.1080/10509670902849038

57. Gagnon NC. Mental Health Screening in Jails. Burnaby, BC: Department of Psychology-Simon Fraser University (2009).

58. Baksheev GN, Ogloff J, Thomas S. Identification of mental illness in police cells: a comparison of police processes, the Brief Jail Mental Health Screen and the Jail Screening Assessment Tool. Psychol Crime Law. (2012) 18:52942. doi: 10.1080/1068316X.2010.510118

59. Grubin D, Carson D, Parsons S. Report on New Prison Reception Health Screening Arrangements: The Results of a Pilot Study in 10 Prisons. Callaghan, NSW: University of Newcastle (2002).

60. Birmingham L, Gray J, Mason D, Grubin D. Mental illness at reception into prison. Crim Behav Mental Health. (2000) 10:77-87. doi: 10.1002/cbm.347

61. Gavin N, Parsons S, Grubin D. Reception screening and mental health needs assessment in a male remand prison. Psychiatr Bulletin. (2003) 27:2513. doi: 10.1017/S095560360000252X

62. Tien G, Ogloff J, Roesch R, Wilson D, Grant F, Mah B. Surrey Pretrial Mental Health Project: Evaluation Report for the Management Committee. Vancouver, BC: British Columbia Forensic Psychiatric Services Commission (1993).

63. Nicholls TL, Lee Z, Corrado RR, Ogloff JR. Women inmates' mental health needs: evidence of the validity of the Jail Screening Assessment Tool (JSAT). Int J Forensic Ment Health. (2004) 3:167-84. doi: 10.1080/14999013.2004.10471205

64. Martin MS, Dorken SK, Wamboldt AD, Wootten SE. Stopping the revolving door: a meta-analysis on the effectiveness of interventions for criminally involved individuals with major mental disorders. Law Hum Behav. (2012) 36:1-12. doi: 10.1037/h0093963

65. Ginsberg Y, Lindefors N. Methylphenidate treatment of adult male prison inmates with attention-deficit hyperactivity disorder: randomised double-blind placebo-controlled trial with open-label extension. Br J Psychiatry. (2018) 200:68-73. doi: 10.1192/bjp.bp.111.0 92940

66. Konstenius M, Jayaram-Lindström N, Guterstam J, Beck O, Philips B, Franck J. Methylphenidate for attention deficit hyperactivity disorder and drug relapse in criminal offenders with substance dependence: a 24-week randomized placebo-controlled trial. Addiction. (2013) 109:4409. doi: 10.1111 /add.12369

67. Ehret W. How to increase medication adherence: what works? Mental Health Clinician. (2013) 2:132973. doi: 10.9740/mhc.n132973

68. Tomlinson MF. A theoretical and empirical review of dialectical behavior therapy within forensic psychiatric and correctional settings worldwide. Int J Forensic Ment Health. (2018) 17:72-95. doi: 10.1080/14999013.2017.1 416003

69. Bradley RG, Follingstad DR. Group therapy for incarcerated women who experienced interpersonal violence: a pilot study. J Trauma Stress. (2003) 16:337-40. doi: 10.1023/A:1024409817437 
70. Johnson JE, Zlotnick C. Pilot study of treatment for major depression among women prisoners with substance use disorder. J Psychiatr Res. (2012) 46:117483. doi: 10.1016/j.jpsychires.2012.05.007

71. Harner $H$, Hanlon AL, Garfinkel $M$. Effect of iyengar yoga on mental health of incarcerated women. Nurs Res. (2010) 59:389-99. doi: 10.1097/NNR.0b013e3181f2e6ff

72. Valentine PV, Smith TE. Evaluating traumatic incident reduction therapy with female inmates: a randomized controlled clinical trial. Res Soc Work Pract. (2001) 11:40-52. doi: 10.1177/104973150101100103

73. Wolff N, Huening J, Shi J, Frueh BC, Hoover DR, McHugo G. Implementation and effectiveness of integrated trauma and addiction treatment for incarcerated men. J Anxiety Disord. (2015) 30:66-80. doi: 10.1016/j.janxdis.2014.10.009

74. Cole KL, Sarlund-Heinrich P, Brown LS. Developing and assessing effectiveness of a time-limited therapy group for incarcerated women survivors of childhood sexual abuse. J Trauma Dissociat. (2007) 8:97121. doi: 10.1300/J229v08n02_07

75. Gussak D. Comparing the effectiveness of art therapy on depression and locus of control of male and female inmates. Arts Psychother. (2009) 36:2027. doi: 10.1016/j.aip.2009.02.004

76. Khalifa N, Saleem Y, Stankard P. The use of telepsychiatry within forensic practice: a literature review on the use of videolink. J Forensic Psychiatry Psychol. (2008) 19:2-13. doi: 10.1080/14789940701560794

77. Burke C, Keaton S. San Diego County's Connections Program Board of Corrections Final Report. San Diego, CA: San Diego Association of Governments (2004).

78. Kesten KL, Leavitt-Smith E, Rau DR, Shelton D, Zhang W, Wagner J, et al. Recidivism rates among mentally ill inmates. J Correction Health Care. (2011) 18:20-8. doi: 10.1177/1078345811421117

79. Morrissey JP, Domino ME, Cuddeback GS. Expedited medicaid enrollment, mental health service use, and criminal recidivism among released prisoners with severe mental illness. Psychiatr Serv. (2016) 67:8429. doi: 10.1176/appi.ps.201500305

80. Angell B, Matthews E, Barrenger S, Watson AC, Draine J. Engagement processes in model programs for community reentry from prison for people with serious mental illness. Int J Law Psychiatry. (2014) 37:490500. doi: 10.1016/j.ijlp.2014.02.022

81. Shaw J, Conover S, Herman D, Jarrett M, Leese M, McCrone P, et al. Critical time Intervention for Severely mentally ill Prisoners (CrISP): a randomised controlled trial. Health Serv Deliv Res. (2017) 5:1-138. doi: 10.3310/hsdr05080

82. Davis L, Williams MV, DeRose K, Steinberg P, Nicosia N, Overton A, et al. Understanding the Public Health Implications of Prisoner Reentry in California: State-of-the-State Report. Santa Monica, CA (2011). doi: 10.7249/MG1165

83. Igoumenou A, Kallis C, Coid J. Treatment of psychosis in prisons and violent recidivism. BJPsych Open. (2015) 1:149-57. doi: 10.1192/bjpo.bp.115.000257
84. Chang Z, Lichtenstein P, Langstrom N, Larsson H, Fazel S. Association between prescription of major psychotropic medications and violent reoffending after prison release. J Am Med Assoc. (2016) 316:1798807. doi: 10.1001/jama.2016.15380

85. Rezansoff SN, Moniruzzaman A, Fazel S, McCandless L, Somers JM. Adherence to antipsychotic medication and criminal recidivism in a Canadian Provincial Offender Population. Schizophr Bull. (2017) 43:100210. doi: 10.1093/schbul/sbx084

86. Jones RM, Manetsch M, Gerritsen C, Simpson AI. Patterns and predictors of reincarceration among prisoners with serious mental illness: a cohort study: Modèles et Prédicteurs de Réincarcération Chez les Prisonniers Souffrant de Maladie Mentale Grave: Une Étude de Cohorte. Can J Psychiatry. (2020) 2020:0706743720970829. doi: 10.1177/07067437209 70829

87. Luciano A, Belstock J, Malmberg P, McHugo GJ, Drake RE, Xie H, et al. Predictors of incarceration among urban adults with co-occurring severe mental illness and a substance use disorder. Psychiatr Serv. (2014) 65:132531. doi: 10.1176/appi.ps.201300408

88. Tyler E, Brockmann B. Returning home: incarceration, reentry, stigma and the perpetuation of racial and socioeconomic health inequity. J Law Med Ethics. (2017) 45:545-57. doi: 10.1177/10731105177 50595

89. Billen C, Schulte-Ostermann MA, Huchzermeier C. Clinical Global Impression - corrections (CGI-C) - deutsche Übersetzung. Forensische Psychiatrie Psychologie Kriminologie. (2020) 14:32835. doi: 10.1007/s11757-020-00599-9

Conflict of Interest: The authors declare that the research was conducted in the absence of any commercial or financial relationships that could be construed as a potential conflict of interest.

Publisher's Note: All claims expressed in this article are solely those of the authors and do not necessarily represent those of their affiliated organizations, or those of the publisher, the editors and the reviewers. Any product that may be evaluated in this article, or claim that may be made by its manufacturer, is not guaranteed or endorsed by the publisher.

Copyright (C) 2022 Simpson, Gerritsen, Maheandiran, Adamo, Vogel, Fulham, Kitt, Forrester and Jones. This is an open-access article distributed under the terms of the Creative Commons Attribution License (CC BY). The use, distribution or reproduction in other forums is permitted, provided the original author(s) and the copyright owner(s) are credited and that the original publication in this journal is cited, in accordance with accepted academic practice. No use, distribution or reproduction is permitted which does not comply with these terms. 